\title{
Bipolar Electrochemical Measurement of Enantiomeric Excess with Inherently Chiral Polymer Actuators
}

\author{
Serena Arnaboldi, Gerardo Salinas, Giorgia Bonetti, Roberto Cirilli, Tiziana Benincori, \\ and Alexander Kuhn*
}

Cite This: ACS Meas. Au 2021, 1, 110-116

Read Online

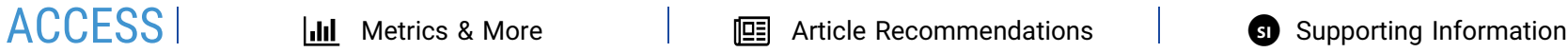

ABSTRACT: Straightforward enantioselective analytical methods are very important for drug safety, considering that in certain cases one of the two enantiomers of a chiral molecule might be harmful for humans. In this work, we propose a simple system for the direct and easy read-out of the enantiomeric excess of 3,4-dihydroxyphenylalanine (DOPA) as a model analyte. A conducting oligomer, i.e. oligo-( $3,3^{\prime}$-dibenzothiophene), bearing inherently chiral features, is electrogenerated on a polypyrrole film. The resulting freestanding hybrid material is used as a wireless enantioselective actuator in a bipolar electrochemical cell. Combining in a single setup two individual actuators with opposite chiral features allows a direct visual read-out of enantiomeric excess, as the bending amplitude of each of the two actuators is directly

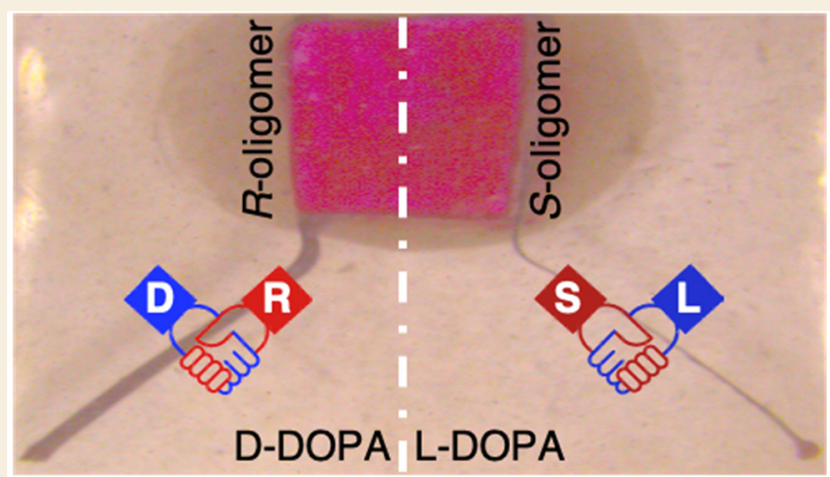
correlated with the concentration of the corresponding stereoisomer of the analyte. Optimization of the experimental parameters results in efficient bending, giving access to the percentage values of the enantiomeric excess in mixtures containing different ratios of the antipodes, thus opening the way to potential applications for chiral in situ analysis.

KEYWORDS: Electrochemical Actuators, Direct Enantiomeric Excess Determination, Inherent Chirality, Absolute Enantiodiscrimination, Bipolar Electrochemistry

\section{INTRODUCTION}

Improvement of life quality has stimulated considerable research in drug safety. To reach this target, sensitive, enantioselective, and fast analytical methods are necessary. ${ }^{1}$ Electrochemical approaches often fulfill this scope. In classic electrochemical transduction, the detector electrode has to be wired to the power supply. Bipolar electrochemistry (BPE) is an interesting alternative, allowing direct electrochemical detection in a wireless way. This method is based on the use of two feeder electrodes and a conducting object (the bipolar electrode), placed in a solution containing supporting electrolyte. The potential difference between the electrolyte and the bipolar electrode drives asymmetric faradaic reactions (reduction/oxidation) at the cathodic and anodic extremities of the object. ${ }^{2,3}$ BPE has been used in the field of electrosynthesis ${ }^{4,5}$ and in photoelectrochemical cells, ${ }^{6}$ electrochemical reactors, and batteries. ${ }^{7}$ Recently, BPE has gained considerable attention for analytical purposes such as preconcentration, ${ }^{8,9}$ separation, ${ }^{10,11}$ electrochemical sensing, $^{12,13}$ and optical detection. ${ }^{14,15}$ For example, Klett et al. coupled bipolar electrochemistry with capillary electrophoresis for the potentiostat-less detection of an iron-based redox couple. ${ }^{12}$ In addition, BPE can be employed in combination with impedance spectroscopy ${ }^{13}$ and spectroelectrochemistry ${ }^{16}$ to develop new sensors. Among all of these methods, characterized by optical or electrochemical readouts of the transduced information, mechanical deformation of a wireless actuator is an extremely easy way to visualize the occurrence of redox processes in a straightforward way. ${ }^{17-23}$ Therefore, if the mechanical deformation, occurring during the bipolar experiment, is combined with chiral recognition, the concept can contribute to the development of new analytical techniques with an added value.

Bipolar electrochemical processes endowed with enantioselectivity have been previously proposed. ${ }^{24,25}$ A chiral imprinted mesoporous platinum film was combined with polypyrrole in a hybrid material, for which the preferential reaction with one enantiomer was detected by a differential electromechanical deformation of the polymer. The relative difference in bending was used as a sign of enantioselectivity, as it is based on a difference in local current intensity. ${ }^{26}$ However, a discrim-

Received: May 26, 2021

Published: July 19, 2021 
ination only in terms of current amplitude has some limitations, e.g., the impossibility to distinguish between two enantiomers of the same molecule if they coexist in the same solution. $^{27,28}$ Thus, it would be preferable if two enantiomers were also able to induce thermodynamic signal differences. In this context, an alternative approach was recently proposed, involving a particular type of chirality, intrinsic to the whole material, named inherent chirality. Compounds endowed with inherent chirality are characterized by the presence of an atropoisomeric scaffold or a helix unit. Furthermore, since inherently chiral molecules are endowed with $C_{2}$ symmetry, ${ }^{29,30}$ they have free homotopic positions, suitable for oligomerization, guaranteeing the full regioregularity of the synthesized macromolecular materials. Circular dichroism experiments demonstrated that inherent chirality is fully transferred from the monomer to the oligomer.

When this kind of compound is electrochemically deposited in an enantiopure form on a bulk electrode and tested in an electrochemical setup in the presence of chiral drugs, thermodynamically different voltammetric results are obtained. Well-defined signals with different peak potentials for each enantiomer of the chiral probe can be observed. The potential shift is due to energetically different situations for the two enantiomers on an inherently chiral electrode surface. ${ }^{31}$ This discrimination power of such chiral electrode surfaces has also been confirmed in the presence of unbalanced mixtures of antipodes of chiral drugs, with the possibility to determine enantiomeric excess by recording the voltammetric signal. ${ }^{29,31}$

An analog concept has also been used in combination with bipolar electrochemistry. ${ }^{32,33}$ The synergy between inherent chirality and the electromechanical proprieties of polypyrrole $(\text { Ppy })^{34-37}$ enabled the development of a bipolar electroactuator with a chirality-dependent on-off behavior. The enantiopure inherently chiral oligomer was deposited at one extremity of a Ppy strip and used as a bipolar electrode, reacting differently, with a yes-no response to the presence of the two enantiomers of a redox-active chiral analyte. The observed actuation is proportional to the concentration of the "good" enantiomer, whereas its antipode does not induce any actuation. In the present work, we further explore this concept of chiral bipolar electrochemistry by developing an on-off sensor able to detect directly and quantitatively the enantiomeric excess in unbalanced mixtures containing different ratios of the enantiomers of a model analyte ( $\mathrm{L}$ - or D-3,4-dihydroxyphenylalanine, L- or D-DOPA).

\section{EXPERIMENTAL SECTION}

\section{Synthesis of the Hybrid Films}

For the electrosynthesis of Ppy, a solution of pyrrole monomer ( 0.2 $\mathrm{M}$, Sigma-Aldrich) was dissolved in an aqueous dodecylbenzenesulfonate (0.25 M, Sigma-Aldrich) solution. Two gold-coated glass slides were positioned parallel in a beaker filled with $12 \mathrm{~cm}^{3}$ of this solution. These gold-coated glass slides were used as a working and counter electrode, whereas $\mathrm{Ag} / \mathrm{AgCl}(3 \mathrm{M} \mathrm{KCl})$ was the reference electrode. A fixed current of $4 \mathrm{~mA}$ was applied for $1.5 \mathrm{~h}$ for the polymerization of pyrrole. After polymerization, the Ppy film was washed with water, dried, and peeled off to be used as a support for the further oligomerization of the chiral monomer, i.e., 2,2'-bis[2-(5,2'bithienyl)]-3,3'-bithianaphthene $\left(\mathrm{BT}_{2} \mathrm{~T}_{4}\right)$ (Figure $\left.1 \mathrm{a}\right)$. The electrosynthesis of enantiopure oligo- $(S)-\mathrm{BT}_{2} \mathrm{~T}_{4}$ and oligo- $(R)-\mathrm{BT}_{2} \mathrm{~T}_{4}$ was carried out with the polypyrrole substrate acting as the working electrode in a small beaker containing $5 \mathrm{~cm}^{3}$ of $0.1 \mathrm{M}$ lithium perchlorate $\left(\mathrm{LiClO}_{4}\right)$ solution in acetonitrile $(\mathrm{MeCN})$ and the $(R)$ - or $(S)$-enantiopure monomers at $5 \mathrm{mM}$ concentration. A platinum grid

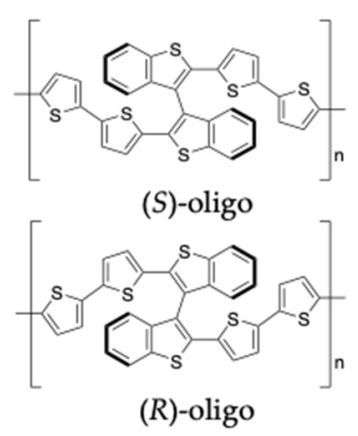

A

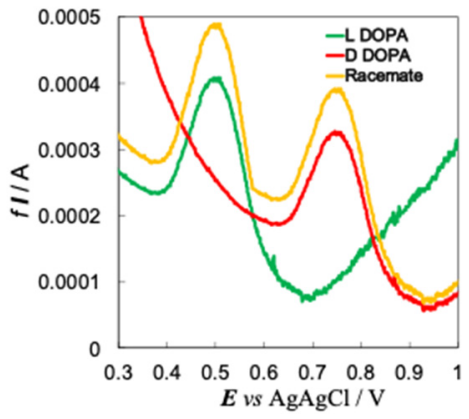

B
Figure 1. (A) Chemical structures of the two enantiomers of the $\mathrm{BT}_{2} \mathrm{~T}_{4}$ oligomers. (B) Differential pulse voltammetry in an aqueous solution of $0.2 \mathrm{M} \mathrm{LiClO}_{4}$ supporting electrolyte for the enantioselective electro-oxidation of $\mathrm{L}$ - and D-DOPA $(5 \mathrm{mM})$, green and red lines, respectively, and of a DOPA racemate, yellow line ( $\mathrm{L}-+$ $\mathrm{D}$-DOPA $=10 \mathrm{mM})$ with an oligo- $(S)-\mathrm{BT}_{2} \mathrm{~T}_{4}$-modified freestanding Ppy film.

and a $\mathrm{Ag} / \mathrm{AgCl}$ electrode were used as counter and reference electrodes, respectively. Considering that the total length of the selfstanding film was around $2 \mathrm{~cm}$, only a fraction of $1 \mathrm{~cm}$ of the Ppy has been dipped in the solution for further modification with the oligomers. Oligo- $(S)-\mathrm{BT}_{2} \mathrm{~T}_{4}$ and oligo- $(R)-\mathrm{BT}_{2} \mathrm{~T}_{4}$ were synthesized by chronopotentiometry at a fixed current value of $2 \mathrm{~mA}$ for $40 \mathrm{~min}$. After the deposition, the oligo-(3,3'dibenzothiophene)-polypyrrole hybrid films were cut into strips of different geometry to be used as chiral sensors. The PPy film has a thickness comprising between 60 and $70 \mu \mathrm{m}$ and the enantiopure thin oligomer layer of about $1 \mu \mathrm{m}$. Experimental details of the elaboration of the hybrid films are illustrated in Scheme S1.

\section{Differential Pulse Voltammetry (DPV) Experiments}

DPV experiments were carried out in an electrochemical cell, containing the enantiomers of L- or D-DOPA $(5 \mathrm{mM})$ dissolved in an aqueous $0.2 \mathrm{M} \mathrm{LiClO}_{4}$ solution. A platinum grid and a $\mathrm{Ag} / \mathrm{AgCl}$ electrode were used as counter and reference electrodes, respectively. The hybrid electrode, composed of an oligo- $(S)$ - or oligo- $(R)-\mathrm{BT}_{2} \mathrm{~T}_{4}$ layer, deposited on a freestanding Ppy film was used as the working electrode. The optimized DPV parameters for recording the voltammetric signals of $\mathrm{L}-$ or $\mathrm{D}-\mathrm{DOPA}$ were as follows: step potential $10 \mathrm{mV}$, modulation amplitude $60 \mathrm{mV}$, modulation time $40 \mathrm{~ms}$, and interval time $200 \mathrm{~ms}$.

\section{Enantiomeric Excess Measurements}

For the enantiomeric excess determination by bipolar electrochemistry, enantiopure $(R)$ - or $(S)$-oligo-(3,3'-dibenzothiophene)polypyrrole hybrid films ( 15 or $17 \mathrm{~mm}$ long and with 0.06 or 0.03 $\mathrm{mm}^{2}$ cross-sectional area) were fixed on the two opposite sides of a rectangular-shaped support in the center of the bipolar cell. The fraction of the strip that was fixed at the support was $8 \mathrm{~mm}$ long in all cases. Two graphite feeder electrodes were positioned at the extremities of the cell $(5 \mathrm{~cm}$ apart, Figure S1). An aqueous $0.2 \mathrm{M}$ $\mathrm{LiClO}_{4}$ solution was used as supporting electrolyte to provide a sufficient amount of ions for charge compensation in the conducting polymer during the bipolar actuation in the presence of unbalanced mixtures of the two antipodes of DOPA. The analyzed D- DOPA/LDOPA molar concentration ratios were $0: 10,3: 7,5: 5$, and $7: 3$ by maintaining the total concentration of D- + L-DOPA constant $(10$ $\mathrm{mM}$ ). The ratios 5:0 and 0:5 refer to solutions for which only LDOPA or D-DOPA were added separately at a concentration of 5 $\mathrm{mM}$. The degree of bending of the actuator was recorded using a macroscope (LEICA Z16 APO) in video mode. Movie data treatment was carried out with ImageJ software.

\section{Polypyrrole Resistance Measurements}

The resistance of the Ppy cantilevers was measured by means of a digital multimeter. Four different strips of the hybrid materials were 
cut with different geometries: (i) length of the Ppy strip $9 \mathrm{~mm}$ (total length $17 \mathrm{~mm}$ ), cross-sectional area of $0.06 \mathrm{~mm}^{2}$; (ii) length of the Ppy strip $9 \mathrm{~mm}$ (total length $17 \mathrm{~mm}$ ), cross-sectional area of 0.03 $\mathrm{mm}^{2}$; (iii) length of the Ppy strip $3 \mathrm{~mm}$ (total length $11 \mathrm{~mm}$ ), crosssectional area of $0.03 \mathrm{~mm}^{2}$; (iv) length of the Ppy strip $9 \mathrm{~mm}$ (total length $17 \mathrm{~mm}$ ), cross-sectional area of $0.06 \mathrm{~mm}^{2}$, with nail varnish covering the Ppy cantilever starting $3 \mathrm{~mm}$ away from the support until the end of the strip. The thickness of the films was in all four cases around $60 \mu \mathrm{m}$. Bipolar measurements were carried out in this case by fixing only one strip of the hybrid material on the support, positioned in an aqueous $5 \mathrm{mM}$ L-DOPA and $0.2 \mathrm{M} \mathrm{LiClO}_{4}$ solution. The applied electric field was the same in all four cases $\left(0.6 \mathrm{~V} \mathrm{~cm}^{-1}\right)$. The degree of actuation was recorded using a macroscope (LEICA Z16 $\mathrm{APO}$ ) in video mode. Movie data treatment was carried out with ImageJ software.

\section{RESULTS AND DISCUSSION}

The experimental setup, including the actuation mechanism, is illustrated in Scheme 1 and Figure S1. The bipolar device

Scheme 1. Schematic Illustration of the Setup Used for the Wireless Enantiomeric Excess Detection of Mixtures of $L^{-}$ and D-DOPA before and after Applying the Electric Field ${ }^{a}$

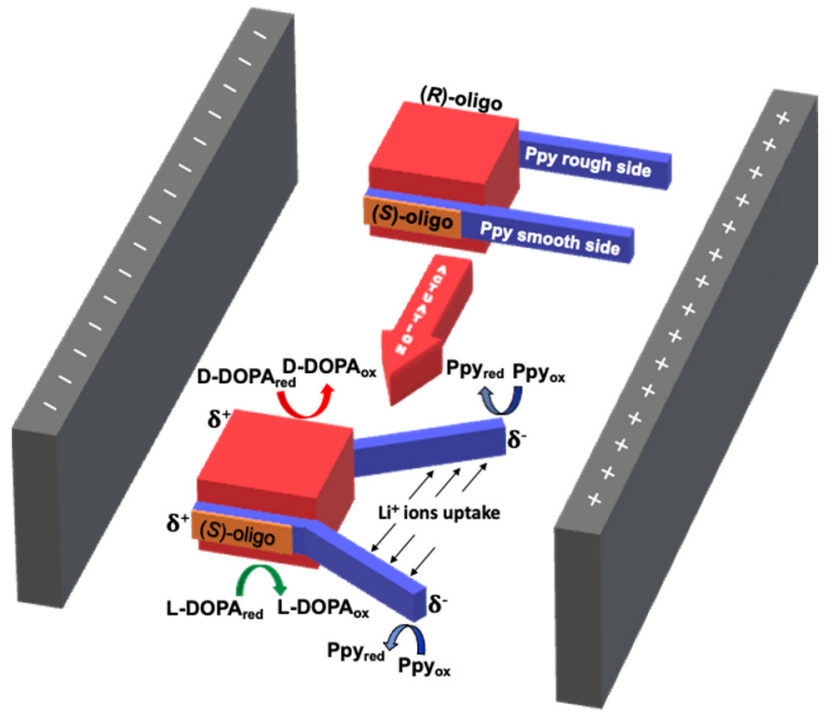

${ }^{a}$ The red cube represents the inert support on which the hybrid strips are fixed. The orange part represents the $(S)-\mathrm{BT}_{2} \mathrm{~T}_{4}$ oligomer (the corresponding counterpart modified with $(R)-\mathrm{BT}_{2} \mathrm{~T}_{4}$ oligomer is hidden at the backside of the inert support), whereas blue symbolizes the Ppy film. The actuation mechanism involves the selective oxidation of L- and D-DOPA on the two enantiopure oligomermodified extremities and the reduction of Ppy, accompanied by bending induced by the cation uptake.

consists of two individual hybrid polymer strips, facing each other and being fixed on an inert support (red). The polymer strips are composed of enantiopure $(R)$ - or $(S)$-oligomers (orange) electrodeposited on Ppy (blue). Under the influence of an electric field of $0.6 \mathrm{~V} \mathrm{~cm}^{-1}$, the composite bipolar electrodes are polarized with respect to the surrounding solution, generating $\delta^{+}$and $\delta^{-}$extremities. At the $\delta^{+}$side, electro-oxidation of L- or D-DOPA can take place on the enantiopure oligomer surface. At the $\delta^{-}$extremity, Ppy is reduced, accompanied by the insertion of cations, in order to maintain electroneutrality, leading to a swelling of the polymer. The electrosynthesis of the Ppy strip was carried out on a flat electrode, meaning that, after peeling off the freestanding film from the substrate, the film presents one smooth face (the one in contact with the electrode surface) and a rough face (the one directed toward the electrolyte). ${ }^{18}$ The chiral oligomer was deposited on the smooth part of the polypyrrole. During the reduction of Ppy, cations enter the polymer preferentially at the rough face of the film. Since in the present setup the rough side of the film is facing inward, the uptake of cations will induce a significant bending of the cantilever in the opposite outward direction.

The inherently chiral oligomer employed in the selective electro-oxidation is a molecule with a bibenzothiophene as the central core (Figure 1A). The high racemization barrier allows separating the molecule into enantiomers, which are stable at room temperature.

As a preliminary test, we performed enantiorecognition measurements by means of differential pulse voltammetry, using as a working electrode a hybrid freestanding film constituted by Ppy and a $(S)$-oligomer layer, in the presence of either individual $5 \mathrm{mM} \mathrm{L}$ - or D-DOPA solutions or a DOPA racemate solution (total concentration of $10 \mathrm{mM}$ ). The DPV experiments show a clear separation in terms of oxidation potential when comparing the two enantiomers of the analyte (Figure 1B). The voltametric signals of $\mathrm{L}^{-}$and D-DOPA, when analyzed separately, show a peak-to-peak separation of around $300 \mathrm{mV}$. L-DOPA (green line) is preferentially oxidized by $(S)$ $\mathrm{BT}_{2} \mathrm{~T}_{4}$, and consequently, the reaction of the $\mathrm{D}$ analyte (red line) is less favorable. The specular experiment employing $(R)$ $\mathrm{BT}_{2} \mathrm{~T}_{4}$ as a chiral selector is illustrated in Figure S2. The yellow line represents the oxidation obtained on the $(S)$-oligomer when the racemate is present in solution. This illustrates the possibility to detect and discriminate both DOPA enantiomers, even when they are present simultaneously in the solution.

Before proceeding with the enantiomeric excess detection by bipolar electrochemistry, the operating conditions were optimized by employing hybrid film strips with different geometries in order to maximize the bending of Ppy and to find an easy and intuitive way to read-out the enantiomeric excess. A system with only one strip of the hybrid film (the bipolar electrode) has been prepared, composed of Ppy and $(S)$-oligomer, fixed to the red support (Figure 2), and placed in the center of a cell containing an aqueous solution of $5 \mathrm{mM} \mathrm{L}$ DOPA in $0.2 \mathrm{M} \mathrm{LiClO}_{4}$. An electric field of $0.6 \mathrm{~V} \mathrm{~cm}^{-1}$ was applied for recording the actuation of the modified Ppy cantilever. Three actuators with different geometric features have been prepared: (A) length of the free Ppy strip $9 \mathrm{~mm}$ (total length $17 \mathrm{~mm}$ ), cross-sectional area: $0.06 \mathrm{~mm}^{2}$; (B) length of the free Ppy strip $9 \mathrm{~mm}$ (total length $17 \mathrm{~mm}$ ), crosssectional area: $0.03 \mathrm{~mm}^{2}$; (C) length of the free Ppy strip 3 $\mathrm{mm}$ (total length $11 \mathrm{~mm}$ ), cross-sectional area: $0.03 \mathrm{~mm}^{2}$. The thickness of the film was identical $(60 \mu \mathrm{m})$ in all three cases.

Figure 2 illustrates schematically the bending of the bipolar cantilever (left column) together with a superposition of the initial and last frame of a video recording of the bending experiments (right column). The bending behavior of the Ppy cantilever strongly depends on its geometry, which is most likely related to its internal resistance. For a long cantilever (9 $\mathrm{mm}$ ) with a rather high cross-sectional area of $0.06 \mathrm{~mm}^{2}$, the resistance of the polymer is $1.3 \mathrm{k} \Omega$ and the bending occurs like in a classic bipolar setup, as illustrated in Figure $2 \mathrm{~A}^{17-19}$ However, when the strip has the same length $(9 \mathrm{~mm})$ but a smaller cross-sectional area $\left(0.03 \mathrm{~mm}^{2}\right)$, the resistance of Ppy is $4.8 \mathrm{k} \Omega$, and the actuation occurs only along the first $3 \mathrm{~mm}$ of the Ppy cantilever, leaving the remaining part of the polymer 

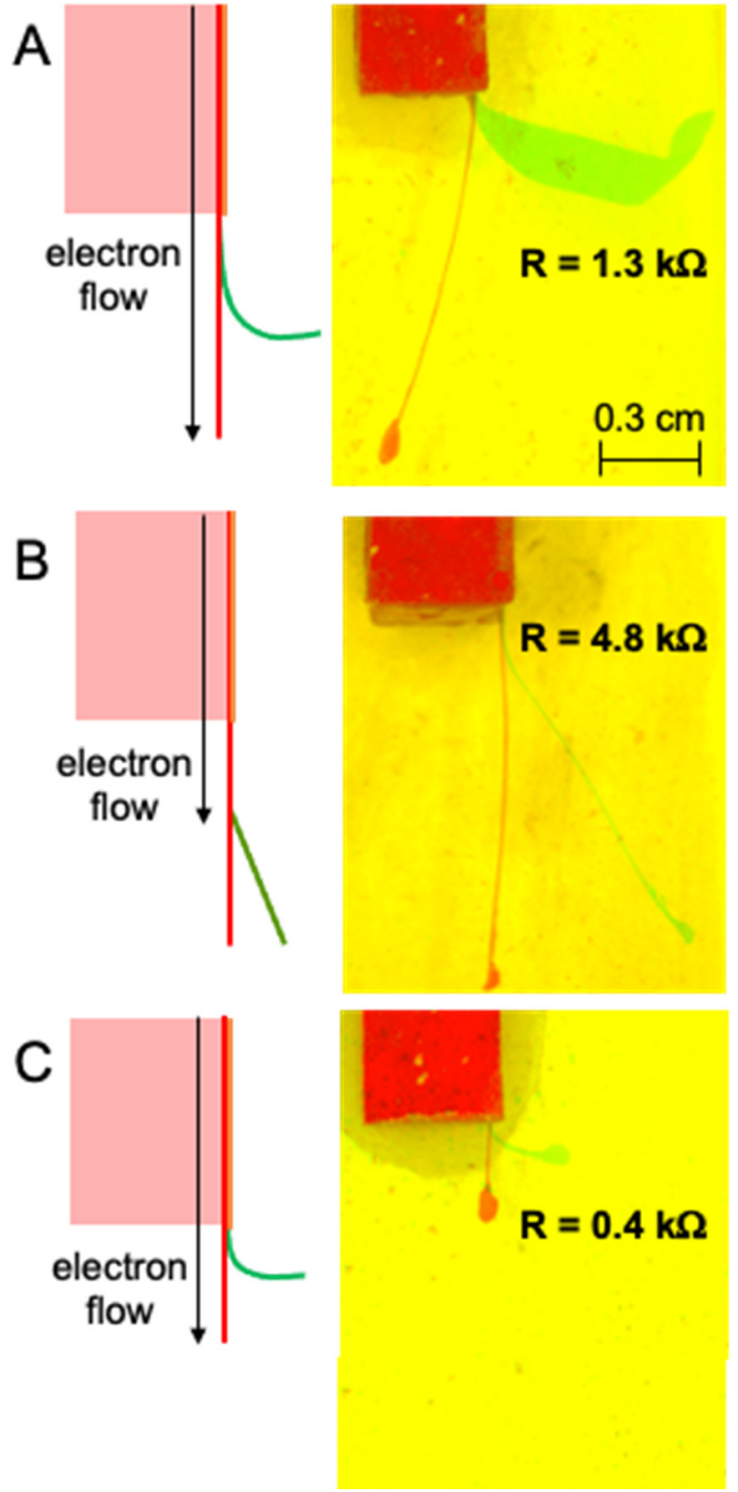

Figure 2. Left column shows the schematic bipolar actuation. Only one hybrid film is employed as a bipolar electrode with different geometrical features of the Ppy cantilever: (A) length $9 \mathrm{~mm}$, crosssectional area of $0.06 \mathrm{~mm}^{2}$; (B) length $9 \mathrm{~mm}$, cross-sectional area of $0.03 \mathrm{~mm}^{2}$; (C) length $3 \mathrm{~mm}$, cross-sectional area of $0.03 \mathrm{~mm}^{2}$. The thickness is in all three cases $60 \mu \mathrm{m}$. The red line represents the cantilever in the initial position. The green line represents the Ppy in the final maximum position after the actuation. In the right column, a superposition of the initial and last video frames related to the experiments are depicted. The bipolar electrodes were placed in an aqueous solution of $5 \mathrm{mM} \mathrm{L}$-DOPA in $0.2 \mathrm{M} \mathrm{LiClO}_{4}$. Resistance values of the Ppy cantilever were measured before the electric field was switched on.

straight (Figure 2B). According to the general equation that correlates the resistance values to the geometrical parameters and the resistivity of an object $(R=(\rho L) / A$, where $\rho$ is the resistivity of the material, $L$ the length of the object, and $A$ its cross-sectional area), we can observe an increase of the resistance as the value of the cross-sectional area decreases. Thus, the electrons generated from the selective oxidation of DOPA at the $\delta^{+}$extremity have to overcome this resistance in order to react at the opposite extremity of the Ppy cantilever. For a too high resistance, the potential drop across the resistance prevents the reduction reaction to occur at the end of the cantilever, and the electrons react instead preferentially at the beginning of the freestanding film. This results in a straight part of the Ppy strip, indicating that this section is not electroactive.

To confirm this hypothesis, the experiment depicted in Figure $2 \mathrm{C}$ has been performed. A strip with the same crosssectional area as the one of Figure $2 \mathrm{~B}\left(0.03 \mathrm{~mm}^{2}\right)$, but with a shorter cantilever $(3 \mathrm{~mm})$, was used. The resistance value is 0.4 $\mathrm{k} \Omega$, in agreement and as expected for the shorter Ppy strip. Actuation occurs in the classic way since the small potential drop does not prevent the electrons from reacting at the end of the hybrid film. To mimic the behavior of the cantilever in Figure 2B, but with the same geometrical features as the one of Figure 2A, a Ppy hybrid film with a length of $9 \mathrm{~mm}$ and a cross-sectional area of $0.06 \mathrm{~mm}^{2}$ has been prepared, covering the strip with nail varnish starting $3 \mathrm{~mm}$ away from the support until the end. In this way, the electron flow through this part of the polymer is inhibited, and bending occurs only in the first part of the Ppy strip (Figure S3).

After these preliminary optimization experiments, bipolar electrochemistry was used for detecting the enantiomeric excess. In Figure 3, each sensor is constituted by two individual

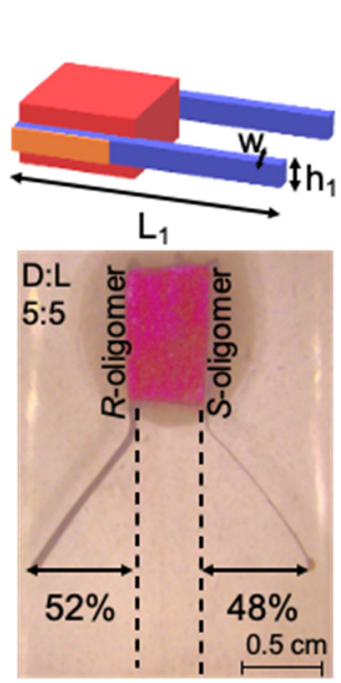

A

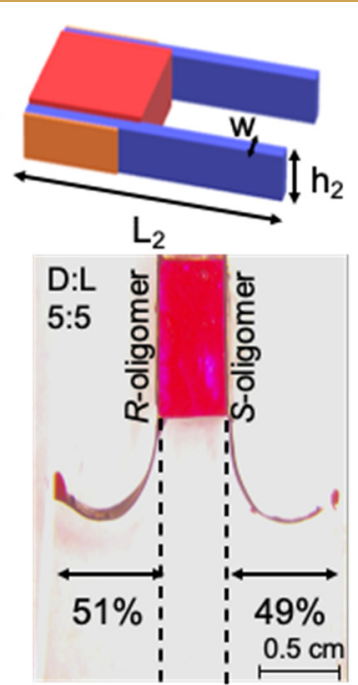

B
Figure 3. Testing relative bipolar actuation with a double cantilever device. In column A, a 3D scheme of the bipolar device illustrates the geometry of a typical setup composed of two cantilevers with a crosssectional area of $0.03 \mathrm{~mm}^{2}\left(L_{1}=17 \mathrm{~mm}, h_{1}=0.5 \mathrm{~mm}, w=60 \mu \mathrm{m}\right)$. In column $\mathrm{B}$, the cross section of $0.06 \mathrm{~mm}^{2}$ allows addressing the full length of the bipolar electrodes due to a smaller resistance of the polymer strips, inducing full bending. The bipolar device is constituted by two strips of Ppy $+(R)$-oligomer and Ppy $+(S)$ oligomer, fixed on the support and placed in a racemic solution of 5 $\mathrm{mM} \mathrm{L}$-DOPA + $5 \mathrm{mM}$ D-DOPA in $0.2 \mathrm{M} \mathrm{LiClO}_{4}$.

strips of the composite films, Ppy $+(S)$-oligomer and Ppy + $(R)$-oligomer, fixed on the red support and placed in an aqueous solution of $10 \mathrm{mM}$ DOPA racemate and $0.2 \mathrm{M}$ $\mathrm{LiClO}_{4}$.

In the experiment illustrated in column $\mathrm{A}$, the Ppy cantilevers have a length of $9 \mathrm{~mm}$ (total length $17 \mathrm{~mm}$ ) and a cross-sectional area of $0.03 \mathrm{~mm}^{2}$, whereas in column $\mathrm{B}$, the strips have the same length and width as in case A but different heights, causing an increase in cross-sectional area $\left(0.06 \mathrm{~mm}^{2}\right)$. 
After an electric field of $0.6 \mathrm{~V} \mathrm{~cm}^{-1}$ is applied, the cantilevers bend in both cases with a final amplitude that can be directly correlated with the enantiomeric ratio of the solution. Since the analyzed mixture is a racemate, the actuation of the Ppy film is in both cases symmetric with respect to the barycenter of the red support. More precisely, the $(R)$-oligomer-modified arm selectively oxidizes D-DOPA, and the $(S)$-oligomermodified part exclusively reacts with L-DOPA. However, the type of bending is different for columns $\mathrm{A}$ and $\mathrm{B}$, due to the different cross-sectional areas (vide supra). Even though the bending amplitude can be converted in both cases into identical enantiomeric ratios $(\sim 50: 50)$ by measuring the relative deflection of the two arms, the bending obtained for $\mathrm{A}$ seems to be more convenient to determine enantiomeric excess in a straightforward way (Video S1). This holds not only for the case of a racemate but also in other cases when the ratio between the two enantiomeric probes is different. Since bending occurs only in the first part of the strip, one can easily calculate the percentage of actuation by measuring the maximum distance between the left side of the red support and the final position of the extremity of the hybrid film modified with the $(R)$-oligomer; the same procedure can also be used for the right side where the $(S)$-hybrid strip is attached. Enantiomeric excess values can then be calculated by dividing the difference between the individual maximum distances by the total deflection of the two polymer arms.

Based on these findings, we decided to use for all subsequent experiments the cantilever geometry of Figure 3A. In particular, the following molar ratios between D-DOPA and L-DOPA were analyzed: $0: 10,3: 7,5: 5$, and $7: 3$ by maintaining constant the total concentration of D- + L-DOPA (10 mM). In principle, lower concentrations might also be used; however, below $2 \mathrm{mM}$, bending is less well pronounced. ${ }^{32}$ For the case of 5:0 and 0:5, L-DOPA and D-DOPA were analyzed separately in $5 \mathrm{mM}$ solutions. The corresponding measured enantiomeric excess values are indicated in Figure 4. They are in very good agreement with the relative concentrations of L- and D-DOPA in each solution. When only L-DOPA is present at $10 \mathrm{mM}$ concentration and the $(R)-\mathrm{BT}_{2} \mathrm{~T}_{4}$ oligomer is deposited on both Ppy strips, no actuation is observed as the $(R)$-oligomer reacts only with D-DOPA. For the 0:5 (and 5:0) molar ratio, the total deflection of the two arms, generally used for the calculation of the enantiomeric excess value, refers only to the one obtained for the arm which is bending. For the racemic solution, the actuation of the two cantilevers is perfectly specular with respect to the barycenter of the red support. Similarly, the result obtained with a 3:7 molar ratio is a mirror image of the one obtained with the $7: 3$ ratio (Video S2). Thus, the optimization of the cantilever geometry allows proposing this approach as a straightforward and simple read-out for the determination of enantiomeric excess, complementary to traditional analytical techniques.

\section{CONCLUSION}

The concept of bipolar electrochemistry was successfully used for the measurement of enantiomeric excess of unbalanced mixtures of L- and D-DOPA as a model analyte. Two hybrid Ppy films, modified with oligomers bearing opposite intrinsic chiral features, were used simultaneously as bipolar electrodes. The actuation efficiency was optimized by adapting the geometry of the freestanding polymer films serving as cantilevers. The respective degree of actuation was found to be directly correlated with the concentration of the chiral
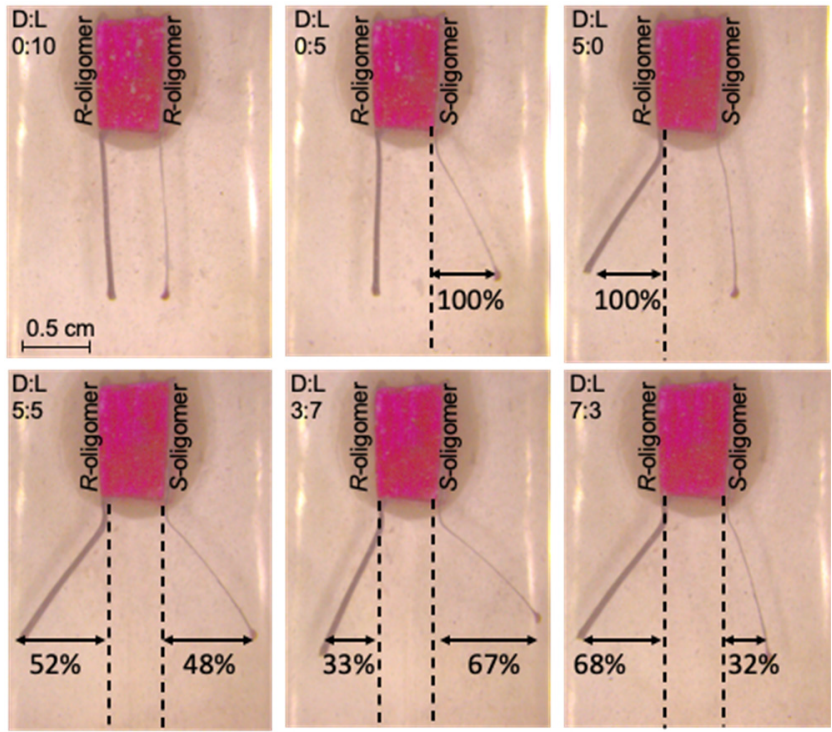

Figure 4. Bipolar electrochemical experiments for the enantiomeric excess determination of solutions with different molar ratios between D- and L-DOPA probes: 0:10, 3:7, 5:5, and 7:3 maintaining constant the total concentration of $\mathrm{L}^{-}+\mathrm{D}-\mathrm{DOPA}(10 \mathrm{mM})$. In the case of 5:0 and 0:5 molar ratios, L-DOPA and D-DOPA were added separately with a concentration of $5 \mathrm{mM}$. The applied electric field is $0.6 \mathrm{~V} \mathrm{~cm}$.

analyte molecules in solution, even when they are present as a mixture. This enables the selective measurement of enantiomeric excess of chiral probes from a qualitative and quantitative point of view. This opens up the perspective to use this technique for the analysis of real samples in the frame of chiral technologies, with the inherent advantage of a bipolar electrochemistry setup that one can build a whole array of such sensing devices, powered by just one pair of feeder electrodes.

\section{ASSOCIATED CONTENT}

\section{Supporting Information}

The Supporting Information is available free of charge at https://pubs.acs.org/doi/10.1021/acsmeasuresciau.1c00011.

Additional experimental scheme, photograph of the experimental setup, differential pulse voltammetry of the $(R)-\mathrm{BT}_{2} \mathrm{~T}_{4}$ oligomer in the presence of DOPA enantiomers, and bipolar behavior of an actuator covered by nail varnish (PDF)

Actuation of a Ppy cantilever in a solution of DOPA racemate $(10 \mathrm{mM})(\mathrm{MP} 4)$

Actuation of a Ppy cantilever in a solution of D- + LDOPA with a 3:7 molar ratio and for the case of the specular 7:3 mixture (MP4)

\section{AUTHOR INFORMATION}

\section{Corresponding Author}

Alexander Kuhn - Université de Bordeaux, CNRS UMR 5255, Bordeaux INP, ENSCBP, 33607 Pessac, France; ○ orcid.org/0000-0002-1962-4863; Email: kuhn@ enscbp.fr

\section{Authors}

Serena Arnaboldi - Université de Bordeaux, CNRS UMR 5255, Bordeaux INP, ENSCBP, 33607 Pessac, France; 
Dipartimento di Chimica, Universita degli Studi di Milano, 20133 Milano, Italy; (0) orcid.org/0000-0002-1981-4659

Gerardo Salinas - Université de Bordeaux, CNRS UMR 5255, Bordeaux INP, ENSCBP, 33607 Pessac, France

Giorgia Bonetti - Dipartimento di Scienza e Alta Tecnologia, Universita degli Studi dell'Insubria, 22100 Como, Italy

Roberto Cirilli - Centro Nazionale per il Controllo e la Valutazione dei Farmaci, Istituto Superiore di Sanità, 00161 Roma, Italy; ○orcid.org/0000-0001-6346-1953

Tiziana Benincori - Dipartimento di Scienza e Alta Tecnologia, Universita degli Studi dell'Insubria, 22100 Como, Italy

Complete contact information is available at:

https://pubs.acs.org/10.1021/acsmeasuresciau.1c00011

\section{Author Contributions}

S.A. designed and performed experiments and wrote and edited the manuscript. G.S. designed the bipolar cell and edited the manuscript. A.K. proposed the research project, provided resources, designed experiments, and edited the manuscript. T.B. designed the inherently chiral monomers. G.B. synthesized the inherently chiral monomers, and R.C. separated the enantiomers of the inherently chiral monomers by chiral HPLC.

\section{Funding}

The work has been funded by the European Research Council (ERC) under the European Union's Horizon 2020 research and innovation program (Grant Agreement No. 741251, ERC Advanced grant ELECTRA). S.A. acknowledges financial support of Università degli Studi di Milano for a partial postdoc scholarship.

Notes

The authors declare no competing financial interest.

\section{ACKNOWLEDGMENTS}

The authors are very grateful for fruitful discussions with Patrizia Mussini.

\section{ABBREVIATIONS}

BPE, bipolar electrochemistry; DPV, differential pulse voltammetry; Ppy, polypyrrol; DOPA, dihydroxyphenylalanine; $\mathrm{LiClO}_{4}$, lithium perchlorate; $\mathrm{MeCN}$, acetonitrile; $\mathrm{BT}_{2} \mathrm{~T}_{4}$, $2^{\prime}$-bis $\left[2-\left(5,2^{\prime}\right.\right.$-bithienyl $\left.)\right]-3,3^{\prime}$-bithianaphthene

\section{REFERENCES}

(1) Kurbanoglu, S.; Unal, M. A.; Ozkan, S. A. Recent Developments on Electrochemical Flow Injection in Pharmaceuticals and Biologically Important Compounds. Electrochim. Acta 2018, 287, 135148.

(2) Koefoed, L.; Pedersen, S. U.; Daasbjerg, K. Bipolar Electrochemistry-A Wireless Approach for Electrode Reactions. Curr. OpinElectrochem. 2017, 2, 13-17.

(3) Loget, G.; Zigah, D.; Bouffier, L.; Sojic, N.; Kuhn, A. Bipolar Electrochemistry: From Materials Science to Motion and Beyond. Acc. Chem. Res. 2013, 46, 2513-2523.

(4) Shida, N.; Zhou, Y.; Inagi, S. Bipolar Electrochemistry: A Powerful Tool for Electrifying Functional Material Synthesis. Acc. Chem. Res. 2019, 52, 2598-2608.

(5) Shida, N.; Inagi, S. Bipolar Electrochemistry in Synergy with Electrophoresis: Electric Field-Driven Electrosynthesis of Anisotropic Polymeric Materials. Chem. Commun. 2020, 56, 14327-14336.
(6) Loget, G.; Li, G.; Fabre, B. Logic Gates Operated by Bipolar Photoelectrochemical Water Splitting. Chem. Commun. 2015, 51, 11115-11118.

(7) Loget, G.; Kuhn, A. Bipolar Electrochemistry in the Nanosciences. In Electrochemistry: Nanosystems Electrochemistry; Wadhawan, J., Compton, R., Eds; RSC Publishing, 2012; Vol. 11, pp $71-103$.

(8) Wei, W.; Xue, G.; Yeung, E. S. One-step Concentration of Analytes based on Dynamic Change in $\mathrm{pH}$ in Capillary Zone Electrophoresis. Anal. Chem. 2002, 74, 934-940.

(9) Anand, R. K.; Sheridan, E.; Hlushkou, D.; Tallarek, U.; Crooks, R. M. Bipolar Electrode Focusing: Tuning the Electric Field Gradient. Lab Chip 2011, 11 (11), 518-527.

(10) Song, H.; Wang, Y.; Garson, C.; Pant, K. Concurrent DNA preconcentration and separation in bipolar electrode-based microfluidic device. Anal. Methods 2015, 7, 1273-1279.

(11) Anand, R. K.; Johnson, E. S.; Chiu, D. T. Negative Dielectrophoretic Capture and Repulsion of Single Cells at a Bipolar Electrode: The Impact of Faradaic Ion Enrichment and Depletion. J. Am. Chem. Soc. 2015, 137, 776-783.

(12) Klett, O.; Nyholm, L. Separation High Voltage Field Driven On-Chip Amperometric Detection in Capillary Electrophoresis. Anal. Chem. 2003, 75, 1245-1250.

(13) Keddam, M.; Nóvoa, X. R.; Puga, B.; Vivier, V. Impedance based Method for Non-Contact Determination of the Corrosion Rate in Buried Metallic Structures. Eur. J. Environ. Civil. Eng. 2011, 15, 1097-1103.

(14) Wu, S.; Zhou, Z.; Xu, L.; Su, B.; Fang, Q. Integrating Bipolar Electrochemistry and Electrochemiluminescence Imaging with Microdroplets for Chemical Analysis. Biosens. Bioelectron. 2014, 53, 148153.

(15) Hu, S.; Chi, X.; Chen, S.; AlTal, F.; Gao, J. Visualizing the Bipolar Electrochemistry of Electrochemically Doped Luminescent Conjugated Polymers. J. Phys. Chem. C 2017, 121, 8409-8415.

(16) Ibañez, D.; Heras, A.; Colina, A. Bipolar Spectroelectrochemistry. Anal. Chem. 2017, 89, 3879-3883.

(17) Gupta, B.; Goudeau, B.; Garrigue, P.; Kuhn, A. Bipolar Conducting Polymer Crawlers Based on Triple Symmetry Breaking. Adv. Funct. Mater. 2018, 28, 1705825.

(18) Gupta, B.; Goudeau, B.; Kuhn, A. Wireless Electrochemical Actuation of Conducting Polymers. Angew. Chem., Int. Ed. 2017, 56, 14183-14186.

(19) Gupta, B.; Afonso, M. C.; Zhang, L.; Ayela, C.; Garrigue, P.; Goudeau, B.; Kuhn, A. Wireless Coupling of Conducting Polymer Actuators with Light Emission. ChemPhysChem 2019, 20, 941-945.

(20) Jansod, S.; Cuartero, M.; Cherubini, T.; Bakker, E. Colorimetric Readout for Potentiometric Sensors with Closed Bipolar Electrodes. Anal. Chem. 2018, 90, 6376-6379.

(21) Cunningham, J. C.; DeGregory, P. R.; Crooks, R. M. New Functionalities for Paper-Based Sensors Lead to Simplified User Operation, Lower Limits of Detection, and New Applications. Annu. Rev. Anal. Chem. 2016, 9, 183-202.

(22) Chow, K.-F.; Mavré, F.; Crooks, J. A.; Chang, B.-Y.; Crooks, R. M. Bipolar electrodes: a useful tool for concentration, separation, and detection of analytes in microelectrochemical systems. J. Am. Chem. Soc. 2009, 131, 8364-8365.

(23) Roark, B.; Tan, J. A.; Ivanina, A.; Chandler, M.; Castaneda, J.; Kim, H. S.; Jawahar, S.; Viard, M.; Talic, S.; Wustholz, K. L.; Yingling, Y. G.; Jones, M.; Afonin, K. A. Fluorescence Blinking as an Output Signal for Biosensing. ACS Sens. 2016, 1, 1295-1300.

(24) Wang, M.; Lin, B.-P.; Yang, H. A plant tendril mimic soft actuator with phototunable bending and chiral twisting motion modes. Nat. Commun. 2016, 7, 13981.

(25) Zhang, P.; Zhou, G.; de Haan, L. T.; Schenning, A. P. H. J. 4D Chiral Photonic Actuators with Switchable Hyper-Reflectivity. Adv. Funct. Mater. 2021, 31, 2007887.

(26) Assavapanumat, S.; Gupta, B.; Salinas, G.; Goudeau, B.; Wattanakit, C.; Kuhn, A. Chiral Platinum-Polypyrrole Hybrid Films 
as Efficient Enantioselective Actuators. Chem. Commun. 2019, 55, 10956.

(27) Kane-Maguire, L. A. P.; Wallace, G. G. Chiral conducting polymers. Chem. Soc. Rev. 2010, 39, 2545-2576.

(28) Gao, M.; Dai, L.; Wallace, G. G. Biosensors Based on Aligned Carbon Nanotubes Coated with Inherently Conducting Polymers. Electroanalysis 2003, 15, 1089-1094.

(29) Sannicolò, F.; Arnaboldi, S.; Benincori, T.; Bonometti, V.; Cirilli, R.; Dunsch, L.; Kutner, W.; Longhi, G.; Mussini, P. R.; Panigati, M.; Pierini, M.; Rizzo, S. Potential-Driven Chirality Manifestations and Impressive Enantioselectivity by Inherently Chiral Electroactive Organic Films. Angew. Chem., Int. Ed. 2014, 53, 26232627.

(30) Arnaboldi, S.; Cauteruccio, S.; Grecchi, S.; Benincori, T.; Marcaccio, M.; Biroli, A. O.; Longhi, G.; Licandro, E.; Mussini, P. R. Thiahelicene-based Inherently Chiral Films for Enantioselective Electroanalysis. Chem. Sci. 2019, 10, 1539-1548.

(31) Arnaboldi, S.; Benincori, T.; Cirilli, R.; Kutner, W.; Magni, M.; Mussini, P. R.; Noworyta, K.; Sannicolò, F. Inherently chiral electrodes: the tool for chiral voltammetry. Chem. Sci. 2015, 6, 1706-1711.

(32) Arnaboldi, S.; Gupta, B.; Benincori, T.; Bonetti, G.; Cirilli, R.; Kuhn, A. Absolute Chiral Recognition with Hybrid Wireless Electrochemical Actuators. Anal. Chem. 2020, 92, 10042-10047.

(33) Arnaboldi, S.; Gupta, B.; Benincori, T.; Bonetti, G.; Cirilli, R.; Kuhn, A. Large Scale Chirality Transduction with Functional Molecular Materials. Chem. Mater. 2020, 32, 10663-10669.

(34) Otero, T. F.; Sansieña, J. M. Soft and Wet Conducting Polymers for Artificial Muscles. Adv. Mater. 1998, 10, 491-494.

(35) Otero, T. F. Biomimetic Conducting Polymers: Synthesis, Materials, Properties, Functions, and Devices. Polym. Rev. 2013, 53, 311-351.

(36) Martinez, J. C.; Otero, T. F.; Jager, E. W. H. ElectrochemoDynamical Characterization of PolypyrroleActuators Coated on Gold Electrodes. Langmuir 2014, 30, 3894-3904.

(37) Garcia-Cordova, F.; Valero, L.; Ismail, Y. A.; Otero, T. F. Biomimetic Polypyrrole based all Three-in-one Triple Layer Sensing Actuators Exchanging Cations. J. Mater. Chem. 2011, 21, 1726517272 . 